PROCEEDINGS OF THE

AMERICAN MATHEMATICAL SOCIETY

Volume 86, Number 1, September 1982

\title{
THERE EXIST ARBITRARILY MANY DIFFERENT DISK KNOTS WITH THE SAME EXTERIOR
}

\author{
L. R. HITT ${ }^{1}$ AND D. W. SUMNERS
}

\begin{abstract}
We prove that, for $n \geq 5$, exteriors of disk knots of $D^{n}$ in $D^{n+2}$ can be exteriors of arbitrarily many different disk knots.
\end{abstract}

1. Introduction. In [H-S], we showed that there are at least three different disk knots of $D^{4}$ in $D^{6}$ with the same exterior, and at least six different disk knots of $D^{n}$ in $D^{n+2}$ with the same exterior for $n \geq 5$. We improve the latter result here by showing that there exist arbitrarily large classes of inequivalent disk knots with the same exterior.

Let $Y$ denote the bounded exterior of a smooth $n$-disk knot. The indeterminacy index $\varsigma(Y)$ is the number of inequivalent $n$-disk pairs having exteriors diffeomorphic to $Y$. We prove the following

THEOREM. Let $n \geq 5$. Given a positive integer $N$, there exists an $n$-disk knot exterior $Y$ with $\varsigma(Y) \geq N$.

This answers Question 1 in [H-S] in the affirmative.

2. The construction. For convenience, we work in the smooth category, although the results hold in the locally flat $P L$ category as well. An $n$-disk knot is a manifold pair $\left(D^{n+2}, f\left(D^{n}\right)\right)$ where $f: D^{n} \rightarrow D^{n+2}$ denotes a proper embedding in which the submanifold $f\left(D^{n}\right)$ intersects $\partial D^{n+2}$ transversely. The exterior $Y$ of an $n$-disk knot is the complement in $D^{n+2}$ of a trivial open 2-disk bundle neighborhood of the submanifold $f\left(D^{n}\right)$. Two disk knots are equivalent if they are diffeomorphic as unoriented pairs, i.e., if there is a diffeomorphism of $D^{n+2}$ onto itself which sends one submanifold onto the other (disregarding orientations).

The construction used in [H-S] to show that $\varsigma(Y)$ can be as large as six was a modification of an example of Kato [Ka, Theorem 4.9]. We recall the construction and further modify it as follows.

Let $G$ be any finitely presented group with $H_{1}(G ; \mathbf{Z})=H_{2}(G ; \mathbf{Z})=0$. If $n+$ $1 \geq 6$, then Kervaire [Ke] has shown that there is a contractible manifold $M^{n+1}$ with $\pi_{1}(\partial M)=G$. Suppose also that $G$ has weight one (i.e., $G$ has an element, called a weight element, whose normal closure is all of $G$ ). Then form the manifold $Y=S^{1} \times M^{n+1}$. We see that $Y$ is a disk knot exterior by attaching a 2-handle $h^{2}$ to $Y$ along the path $t g \in \pi_{1}(\partial Y)=J \times G$, where $g$ is a weight element of $G$, and $t$ is a generator of the infinite cyclic factor $J$. In this case, $t g$ is a weight

Received by the editors June 23, 1981. Presented to the Society, August 21, 1981.

1980 Mathematics Subject Classification. Primary 57Q45.

Key words and phrases. Disk knot, indeterminacy index, weight element.

${ }^{1}$ Research partially supported by a grant from the University of South Alabama Research Committee. 
element of $\pi_{1}(\partial Y)$, and $\left(Y \cup h^{2}, \operatorname{cocore}\left(h^{2}\right)\right)$ is an $n$-disk knot. If now $J \times G$ has many different weight elements, this gives rise to different handle attachments, and possibly inequivalent $n$-disk knots.

To help measure the inequivalency, we call two elements $a, b$ in a group $H$ algebraically distinct if the orbit of the set $\left\{a, a^{-1}\right\}$ under all automorphisms of $H$ is disjoint from the orbit of the set $\left\{b, b^{-1}\right\}$. Then any two algebraically distinct weight elements of $J \times G$ give rise to inequivalent disk knots in this construction. Thus, the proof of the theorem is reduced to finding a suitable class of groups to use in the role of the group $G$.

3. The special linear groups. In [H-S], we used the group $G=\langle a, b| a^{5}=b^{3}=$ $\left.(a b)^{2}\right\rangle=S L(2,5)$ to obtain a group $J \times G$ with three algebraically distinct weight elements, and observed that $J \times G \times G \times G$ contains at least six algebraically distinct weight elements. Here, we use $S L(2, p)$ for $p$ a prime, $p \geq 5$.

Recall that $S L(2, p)$ is its own commutator subgroup (see, e.g., [D, pp. 38-40]), so $H_{1}(S L(2, p))=0$. And, as Gordon [G] points out, $H_{2}(S L(2, p))=0[\mathbf{S}$, p. 95 , Corollary 2]. Furthermore, $Z(S L(2, p))=\{I,-I\}$ where $Z(G)$ denotes the center of the group $G$ and $I$ denotes the identity matrix; and, any noncentral element of $S L(2, p)$ is a weight element (e.g. [R, p. 159]). Thus, any element of the form $t g \in J \times S L(2, p)$, where $t$ generates $J$ and $g$ is not in the center of $S L(2, p)$, is a weight element of $J \times S L(2, p)$. Now let $[a]$ denote the matrix

$$
\left[\begin{array}{cc}
a & 0 \\
0 & a^{-1}
\end{array}\right] \in S L(2, p)
$$

and let $\overline{[a]}$ denote the equivalence class of $[a]$ in the group $\operatorname{PSL}(2, p)$. Since any automorphism of $J \times S L(2, p)$ induces one on

$$
\frac{J \times S L(2, p)}{Z(J \times S L(2, p))} \cong \frac{S L(2, p)}{Z(S L(2, p))} \cong P S L(2, p),
$$

we can show that there are algebraically distinct weight elements in $J \times S L(2, p)$ by showing that their projections in $P S L(2, p)$ are algebraically distinct. But the order of $\overline{[a]} \in P S L(2, p)$ is the same as the order of $a$ in the multiplicative group $\mathbf{Z}_{p}^{*}$ of the field with $p$ elements $\mathbf{Z}_{p}$; and, the order of $\overline{[a]}$ is the same as the order of $\overline{[a]}^{-1}$. Moreover, since $\mathbf{Z}_{p}^{*}$ is cyclic, given any divisor of its order $p-1$, there is an element in $\mathbf{Z}_{p}^{*}$ of that order. The Theorem then follows once it is noted that $\lim \sup \{\tau(p-1) \mid p$ prime $\}=+\infty$ where $\tau(p-1)$ denotes the number of divisors of $p-1$. But this follows from Dirichlet's Theorem, which implies that for any integer $k$, there is a prime of the form $1+k m$.

F. Gonzalez-Acuña [G-A] has pointed out that it follows from Huppert [H, Seite 646, Satz 25.7] that $H_{2}\left(S L\left(2,2^{p-1}\right)\right)=0$ for $p$ prime, $p \geq 5$. Also, $H_{1}\left(S L\left(2,2^{p-1}\right)\right)=0$ since $S L\left(2,2^{p-1}\right)$ is simple. Dirichlet's Theorem can also be used here to show that $\lim \sup \left\{\tau\left(2^{p-1}-1\right) \mid p\right.$ prime $\}=+\infty$.

Thus, either of the classes of groups $S L(2, p), S L\left(2,2^{p-1}\right)$ for $p$ prime, $p \geq 5$, can be used in the role of $G$ in the construction. This completes the proof of the theorem.

As in [H-S], any of the above $n$-disk knot exteriors can be modified by taking the boundary connected sum with an $n$-disk knot having arbitrarily prescribed Alexander polynomial in a single dimension $k(2 \leq k \leq n-1)$ and trivial Alexander 
polynomial elsewhere [Su]. This produces an infinite class of $n$-disk knot exteriors, each having indeterminacy index at least that of the original $n$-disk knot exterior. Thus we have the following

COROllary. Let $n \geq 5$. Given a positive integer $N$, there exist infinitely many homeomorphically distinct $n$-disk knot exteriors, each having indeterminacy index greater than $N$.

NOTE. We have recently learned that F. Gonzalez-Acuña and S. Plotnick (independently) have produced examples with $s(Y)=+\infty$ (private communications).

\section{REFERENCES}

[D] J. Dieudonné, La géométrie des groupes classiques, 2nd ed., Springer, Berlin and New York, 1963.

[G] C. McA. Gordon, Homology of groups of surfaces in the 4-sphere, Math. Proc. Cambridge Philos. Soc. 89 (1981), 113-117.

[G-A] F. Gonzalez-Acuña, personal correspondence.

[H-S] L. R. Hitt and D. W. Sumners, Many different disk knots with the same exterior, Comment. Math. Helv. 56 (1981), $142-147$.

[H] B. Huppert, Endliche Gruppen. I, Springer, Berlin and New York, 1967.

[Ka] M. Kato, Higher dimensional PL knots and knot manifolds, J. Math. Soc. Japan 21 (1969), 458-480.

[Ke] M. Kervaire, Smooth homology spheres and their fundamental groups, Trans. Amer. Math. Soc. 144 (1969), 67-72.

[R] J. J. Rotman, The theory of groups: An introduction, Allyn and Bacon, Boston, Mass., 1965.

[S] R. Steinberg, Lectures on Chevalley groups, Yale Univ. Lecture Notes, 1968.

[Su] D. W. Sumners, Homotopy torsion in codimension two knots, Proc. Amer. Math. Soc. 24 (1970), 229-240.

DEPARTMENT OF MATHEMATICS AND STATISTICS, UNIVERSITY OF SOUTH ALABAMA, MoBILE, AlABAMA 36688

Department of MAThematics, Florida State University, Tallahassee, FLORIDA 32306 